\title{
Résultats De 5ans De Thyroïdectomie Au Service D'orl Et Chirurgie Cervico-Faciale De l'Hôpital National De Niamey (Niger)
}

\author{
Illé $S$. \\ Service ORL et Chirurgie Cervico-Faciale, \\ à l'Hôpital National de Niamey, Niger \\ James Didier $\mathbf{L}$. \\ Saidou A. \\ Service de chirurgie viscérale et digestive, \\ à l'Hôpital National de Niamey, Niger \\ Timi $N$. \\ Service ORL et Chirurgie Cervico-Faciale, \\ à l'Hôpital National de Niamey, Niger \\ Sani $R$. \\ Service de chirurgie viscérale et digestive, \\ à l'Hôpital National de Niamey, Niger
}

doi: 10.19044/esj.2017.v13n21p44 URL:http://dx.doi.org/10.19044/esj.2017.v13n21p44

Abstract

Purpose: To evaluate the results of thyroidectomy in the Oto-RhinoLaryngology and Neck Surgery office of the National Hospital of Niamey in Niger. Materials and methods: we analyzed the epidemiological aspects, the indications, the technics and the evolution of thyroidectomy through a retrospective and descriptive study conducted from January 2010 to December 2015. Results: Thyroidectomy represents 8, 51\% $(n=236 / 2773)$ of the ENT operative activities and an average of 47.2 cases per year for 5 years. Among the patients, there were 15 men and 221 women, a sex ratio of 0.17. The mean age was 37.25 years (extreme 22 and 61 years). The operative indications are dominated by multinodular goiter euthyroid (43.22\%), thyroid nodules (39.40\%), hyperthyroidism (goiter and Basedow) and substernal goiters, respectively, representing $8.90 \%$ and $6.35 \%$. The gestures performed were subtotal thyroidectomy in 56 cases $(23.73 \%)$, complete thyroidectomy in 85 cases $(36.02 \%)$ and lobo-isthmectomy in 95 cases $(40.25 \%)$. The recurrent nerve was searched in 235 cases $(99 \%)$ and found in 215 cases $(91,10 \%)$. It was found 1 case $(0.42 \%)$ of splitting of the left nerve recurrent. 2 cases $(0.84 \%)$ of immediate postoperative hemorrhage was recorded. Morbidity was $0.29 \%$ with 4 cases of definitive unilateral 
recurrent paralysis and 3 cases of transient hypoparathyroidism. No mortality was encountered. Conclusion: The thyroidectomy is a frequent intervention in the ENT and Neck Surgery office of the National Hospital of Niamey. Multinodular goiter remains the first surgical indication. Recurrent and hypoparathyroid morbidity was very low.

Keywords: Multinodular goiter, Thyroidectomy, morbidity, mortality

\section{Résume}

But : Evaluer les résultats des thyroïdectomies au service d'ORL et Chirurgie Cervico-Faciale de l'Hôpital National de Niamey au Niger.

Matériels et méthode: De janvier 2010 à décembre 2015, nous avons analysé les aspects épidémiologiques, les indications, les techniques et l'évolution des thyroïdectomies grâce à une étude rétrospective et descriptive. Résultats: La thyroïdectomie représentait 8,51 \% (n=236/2773) des activités opératoires ORL et une moyenne de 47,2 cas par an pendant 5ans. On notait 15 hommes et 221 femmes, soit un sex- ratio de 0,17 . L'âge moyen était de 37,25 ans (extrêmes 22 et 61 ans). Les indications opératoires sont dominées par le goitre multinodulaire euthyroïdien (43,22\%), les nodules thyroïdiens $(39,40 \%)$, les hyperthyroïdies (goitre et basedow) et les goitres plongeants qui représentaient respectivement $8,90 \%$ et $6,35 \%$ des cas. Les gestes réalisés étaient: une thyroïdectomie subtotale dans 56 cas $(23,73 \%)$, une thyroïdectomie totale dans 85 cas $(36,02 \%)$, une loboisthmectomie dans 95 cas $(40,25 \%)$. Le nerf récurent a été recherché dans 235 cas $(99 \%)$ et trouvé dans 215 cas $(91,10 \%)$.Il a été retrouvé 1 cas $(0,42 \%)$ de dédoublement du nerf récurent gauche. 2 cas $(0,84 \%)$ d'hémorragie postopératoire immédiate ont été enregistrés. La morbidité a été de 0,29\% avec 4 cas de paralysie récurrentielle unilatérale définitive et 3 cas d'hypoparathyroidie transitoire. La mortalité était nulle. Conclusion: La thyroïdectomie est une intervention fréquente en ORL à l'hôpital national de Niamey. Le goitre multinodulaire demeure la première indication chirurgicale. La morbidité récurrentielle et hypoparathyroïdienne a été faible.

Mots-Clés : Goitre multinodulaire, Thyrö̈dectomie, morbidité, mortalité

\section{Introduction}

Les affections thyroïdiennes sont diverses et fréquentes, surtout chez la femme (Monabéka, 2005). Cependant toutes ne relèvent pas de la chirurgie. La Chirurgie reste une option importante dans certaines affections thyroïdiennes en particulier en cas de cancer thyroïdien, de volumineux nodule, de goitre multi nodulaire et d'hyperthyroïdie (Sani, 2010). Bien que ce soient des interventions de pratique courante en Oto-rhino-laryngologie, les thyroïdectomies ne sont toutefois pas dénuées de complications, d'où la 
nécessité de bonnes indications. L'objectif de cette étude est de rapporter les résultats des thyroïdectomies notamment sa fréquence, ses indications, ses aspects techniques et les suites opératoires.

\section{Matériels et Méthode}

Il s'agissait d'une étude rétrospective, descriptive, réalisée au service d'Oto-rhino-laryngologie et de Chirurgie Cervico-Faciale (CCF) de l'Hôpital National de Niamey La période d'étude était du 1er janvier 2010 au 31décembre 2015. Six médecins spécialistes en ORL assuraient les activités chirurgicales ORL pendant la période de l'étude.L'étude a intéressé tous les patients hospitalisés dans le dit service pour thyroidectomie en période d'euthyroïdie. Les strumites et les patients ne répondant pas à ces critères n'étaient pas inclus. La technique opératoire est celle de la chirurgie classique : Le patient sur table opératoire, en décubitus dorsal, la tête en hyperextention avec un billot sous les épaules. L'anesthésie a été générale dans tous les cas. L'abord de la thyroïde a été effectué par une incision curviligne en cravate de KOCHER à deux travers de doigt au-dessus de la fourchette sternale. Le premier temps a visé le relèvement du plan superficiel: la peau, le tissu cellulaire sous-cutané, le muscle peaucier du cou et l'aponévrose cervicale médiane. Le deuxième temps a pénétré la gaine viscérale du cou, découvre le bon plan de clivage, mobilise et extériorise la tumeur avec la ligature-résection du pédicule vasculaire supérieur et des vaisseaux du pôle inférieur. Ce temps recherche et découvre le nerf récurrent, le conserve de même que les parathyroïdes. Enfin suit l'ablation de la tumeur. Le troisième temps a assuré l'hémostase, la mise en place d'un dispositif de drainage et la fermeture en trois plans. Tous ces patients ont fait l'objet d'un examen clinique ORL préopératoire complet y compris une nasofibroscopie et un examen anatomopathologique de la pièce opératoire. L'indication de la thyroïdectomie est retenue sur les données de l'examen clinique et paraclinique (l'échographie thyroïdienne préopératoire, scintigraphie et bilan hormonal). Les supports pour la collecte des données ont été les registres de consultations, les dossiers individuels des patients, les registres de compte rendu opératoire et d'anatomopathologie. Les paramètres étudiés sont d'ordre épidémiologique, clinique, thérapeutique et évolutif. Les paramètres épidémiologiques concernent l'âge, le sexe. Les paramètres cliniques concernent le type de pathologies thyrö̈diennes, les indications opératoires, et la durée moyenne d'hospitalisation. Les aspects thérapeutiques et évolutifs étaient en rapport avec les gestes chirurgicaux exécutés et les suites opératoires à 1 mois. Au delà d'1 mois, le mauvais remplissage des dossiers des patients et la difficulté de suivre les patients, une fois opérés, n'offraient pas la possibilité de disposer des données postopératoires à long terme. 


\section{Résultats}

\section{Données épidémiologiques}

Sur 2773 interventions chirurgicales réalisées en 6 ans, 236 ont concerné la thyroïdectomie, soit une fréquence de $8,51 \%$, et une moyenne de 39,33 cas de thyroidectomie par an. On notait 15 hommes et $221 \mathrm{femmes}$, soit un sex- ratio de 0,17. L'âge moyen était de 37,25 ans (extrêmes 22 et 61 ans). La répartition des patients ayant subi la thyroidectomie par sexe et tranche d'âge est rapportée dans le tableau I. Les 3 cas de cancers thyroïdiens de cette série se retrouvaient chez des sujets de sexe masculin, soit un risque de cancer thyroïdien 5 fois plus élevé chez l'homme que chez la femme.

Tableau I : Sexe et tranche d'âge (en année)

\begin{tabular}{ccccc}
\hline & Masculin & Féminin & Total & Pourcentage(\%) \\
\hline & & & & \\
$16-30$ & 1 & 59 & 60 & 25,43 \\
$31-45$ & 4 & 113 & 117 & 49,57 \\
$46-60$ & 6 & 32 & 38 & 16,10 \\
$>60$ & 4 & 17 & 21 & 8,90 \\
\hline Total & $\mathbf{1 5}$ & $\mathbf{2 2 1}$ & $\mathbf{2 3 6}$ & $\mathbf{1 0 0 \%}$
\end{tabular}

\section{Indications}

Les indications opératoires (Tableau II) sont dominées par le goitre multinodulaire euthyroïdien (43,22\%), les nodules thyroïdiens $(39,40 \%)$, les hyperthyroïdies $(8,90 \%)$ et les goitres plongeants (Figure $\mathrm{n}^{\circ} 1$ ) qui représentent $(6,35 \%)$ des cas.

Tableau II : Indications des thyroïdectomies

\begin{tabular}{llll}
\hline & Effectif & Pourcentage( $\%)$ \\
\hline Nodule thyrö̈dien & 93 & 39,40 \\
Hyperthyroïdies & 21 & 8,90 & \\
-Maladie de Basedow & 08 & 3,40 & \\
-Goitre hyperthyroïdien & 13 & 43,50 & \\
Goitre multinodulaire & 102 & & \\
euthyroïdien & & 6,35 & \\
Goitre plongeant & 15 & 0,85 & \\
Kystes thyroïdiens & 02 & & \\
Cancers thyroïdiens* & 3 & & $\mathbf{1 0 0}$
\end{tabular}


* Carcinomes papillaires de la thyroïde

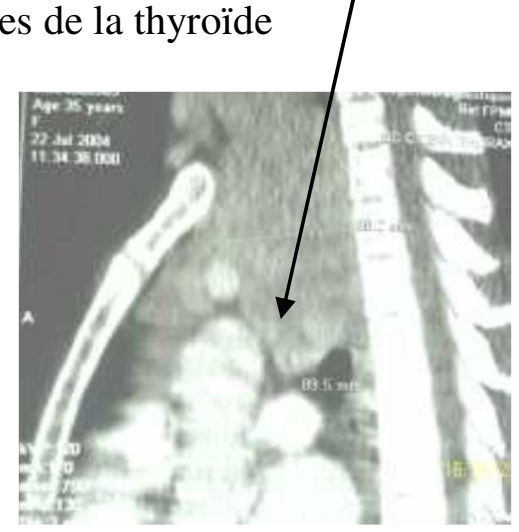

Figure 1 : Coupe scanographique sagittale montrant un goitre plongeant.

\section{Gestes chirurgicaux exécutés}

Le traitement chirurgical était mené par voie cervicale exclusive chez tous les patients et a consisté à une thyroïdectomie subtotale dans 56 cas $(23,73 \%)$, une thyroïdectomie totale dans 85 cas $(36,02 \%)$, une lobo isthmectomie dans 95 cas $(40,25 \%)$. Le nerf récurent a été recherché dans 235 cas $(99 \%)$ et trouvé dans 215 cas $(91,10 \%)$. Dans les vingt un cas $(8,90 \%)$, la recherche était gênée par les saignements rendant difficile la dissection. Il a été retrouvé 1 cas $(0,42 \%)$ de dédoublement du nerf récurent gauche (Figure $\mathrm{n}^{\circ} 2$ ). La ligature et la section de l'artère thyroïdienne inférieure a été réalisée dans 215 cas $(91,10 \%)$ après avoir vu le nerf récurent et dans 21 cas $(8,90 \%)$ sans avoir vu le récurrent. Les parathyroïdes ont été recherchées dans 233 cas $(98,72 \%)$ et retrouvées dans 204 cas $(86,44 \%)$. L'examen histologique définitif a conclu à un carcinome papillaire chez 3 patients de sexe masculin ayant bénéficié d'un complément de curage ganglionnaire (secteur médian et latéral) et adressés au Maroc pour radiothérapie qui n'était pas disponible au Niger.

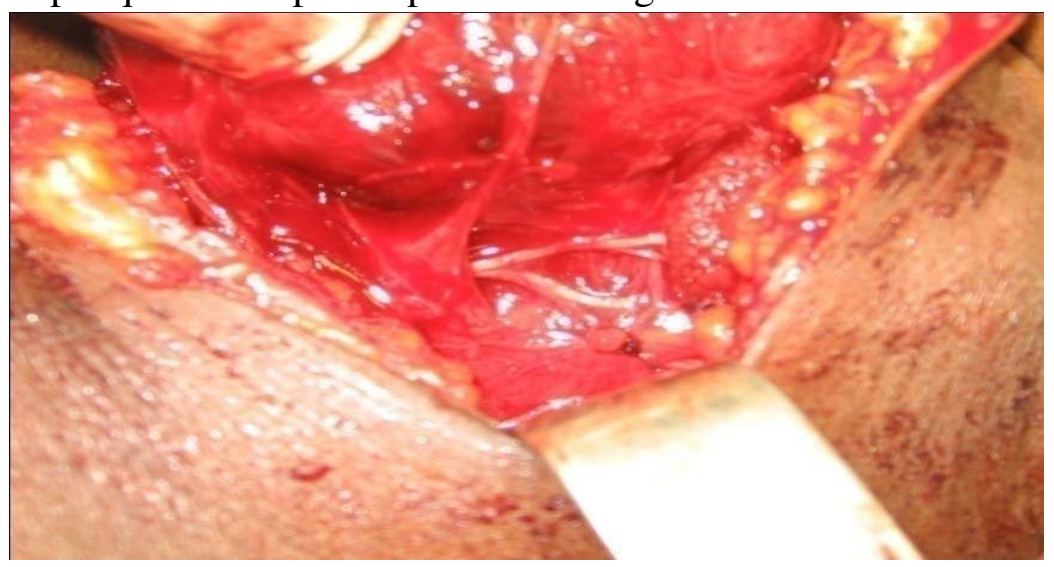

$\underline{\text { Figure } \mathbf{n}^{\circ} \mathbf{2}}$ : Le dédoublement des branches du nerf récurrent gauche. 


\section{Suites opératoires}

La durée moyenne d'hospitalisation pour thyroïdectomie a été de 5 jours avec des extrêmes de 3 et 11 jours. Tous les patients ayant subi une thyroidectomie totale ont bénéficié d'une opothérapie (Lévothyrox) à vie. Les opérés étaient alors revus à J8, J15, et J30.

Les suites opératoires à J30 ont été simples dans 227 cas soit 96.18\% de la série. La mortalité opératoire était nulle. Nous avons enregistré 2 cas $(0,84 \%)$ d'hémorragie post-opératoire immédiate ayant nécessité la transfusion sanguine iso groupe iso rhésus et la reprise chirurgicale pour l'hémostase. La morbidité a été de 0,29\% : 4 cas de paralysie récurrentielle unilatérale définitive et 3 cas d'hypoparathyroidie transitoire à J1 postopératoire, et dont la normalisation est intervenue à partir du quatrième jour à l'aide d'une perfusion de calcium.

\section{Discussion}

En ORL, à l'hôpital national de niamey, la thyroidectomie représente 8,51\% des activités opératoires et une moyenne de 39,33 cas par an pendant 6ans. A Conakry ce geste représente 3,13\% des interventions (Touré, 2006). Dieng et coll (Dieng, 2010) à Abidjan en 2010, ont rapporté 44 à 45 thyroïdectomies par an pendant 9 ans. Ayaché et coll (Ayaché, 2005) à Amiens en France en 2005 ont colligé 735 thyroïdectomies en 12ans, soit 61 cas par an.Spanknebel et coll (Spanknebel, 2006) à New York aux EtatsUnis en 2006 ont effectué 1194 thyrö̈dectomies en 8 ans, soit149 cas par an. Al-Jaradi et coll (Al-Jaradi, 2005) au Yémen en 2005 ont recensé 810 cas en 5ans, soit 162 cas par an. L'âge moyen de 37,25 ans de notre série s'inscrit dans l'intervalle de 34 à 47 ans rapporté dans la littérature (Dieng ,2010 Touré, 2006). La prédominance féminine constatée dans cette étude, est aussi rapportée dans la littérature (Vignikin-Yéhouéssi, 2008). En effet, chez l'homme, la pathologie thyroïdienne est en général plus rare, mais le risque de cancer de la thyroïde est plus élevé chez l'homme que chez la femme (AlJaradi, 2005).

Tout comme dans l'étude réalisée par Ayaché et coll (Ayaché, 2005) en France, Aytac et coll (Aytac, 2005) en Turquie, le goitre multinodulaire venait au premier rang des indications des thyroïdectomies. Les goitres plongeants représentent $6,35 \%$ des thyroïdectomies dans cette série, et 1,9\% dans l'étude réalisée par Sani et coll (Sani, 2010).Placée au troisième rang de nos indications, la maladie de Basedow représente 13\% des indications des thyroïdectomies dans la série de Hill AG et coll (Hill, 2004).

Une thyroidectomie est indispensable dans certains cancers thyroïdiens. La fréquence des thyroïdectomies pour cancers thyroïdiens dans notre étude $(1,28 \%)$ est proche de celle rapportée Aytac et coll (Aytac, 2005 ) en Turquie qui est de 1,3\%. Elle était de 5,39\% dans l'étude réalisée 
par Vignikin-Yéhouéssi (Vignikin-Yéhouéssi, 2008) au Bénin. Tous nos patients ont été opérés sous anesthésie générale. Par contre, dans la série de Spanknebel et coll (Spanknebel, 2006), 79\% de leurs patients ont été opérés sous anesthésie locale, qui selon ces auteurs, a l'avantage d'être moins coûteuse. Nous avions réalisés 56(23,73\%) thyroïdectomies subtotales, 85 $(36,02 \%)$ thyroïdectomies totales et 95(40,25\%) lobo- isthmectomies. Conessa et coll (Conesa, 2000) ont réalisé 71 thyroïdectomies subtotales, 26 thyroïdectomies totales, 52 lobo-isthmectomies et 6 isthmectomies. Dans la série d'étude comme dans plusieurs publications africaines, les thyrö̈dectomies partielles ont été les plus réalisées, tenant compte du niveau socioéconomique bas des patients qui ne peuvent s'assurer un traitement supplétif hormonal onéreux à vie ainsi que l'avantage d'éviter une hypothyroïdie définitive (Dieng, 2010 Sanogo, 2012). Cette attitude n'est pas partagée par certains auteurs Européens (Miccoli,2006 Colak,2004) qui préfèrent plus la thyroidectomie totale qui leur assure une meilleure prévention des récidives dont le traitement est difficile sans donner la garantie de se passer d'une opothérapie substitutive. Le choix de la technique de thyroidectomie dépend de l'expérience de chaque chirurgien et du plateau technique. La technique utilisée par la majorité des auteurs Africains est la thyroidectomie par incision de Kocher et détection visuelle du nerf récurrent. En Europe et aux Etats-Unis, la thyroidectomie par voie endoscopique est en vogue. Néanmoins, quelque soit la technique utilisée (chirurgie classique ou endoscopique), les risques sont toujours présents et ne doivent en aucun cas être minimisés. La morbidité en particulier vis-à-vis du récurrent et des parathyroïdes n'a pas de différence statiquement significative entre ces différentes procédures chirurgicales (Ayaché, 2005). Pour notre part, la thyroidectomie par incision de Kocher a été notre option. La dissection du nerf laryngé inférieur nous a permis de découvrir certaines variations telles que le dédoublement du nerf récurrent à gauche $(0,42 \%)$. Lubrena et al (Lubrena, 2002) dans une étude sur la recherche du nerf récurrent au cours d'une lobectomie, a trouvé fréquemment des bifurcations extra laryngées du récurrent dans environ $20 \%$ des cas. Au lieu de constituer un tronc nerveux unique isolé le nerf est souvent divisé avant son croisement avec l'artère thyroïdienne inférieure. Il donne ainsi des branches extra laryngées, œsophagiennes, pharyngiennes ou trachéales (Lubrena, 2002).

Nous avons enregistré 2 cas $(0,84 \%)$ d'hémorragie post-opératoire immédiate ayant nécessité la transfusion sanguine iso groupe iso rhésus et la reprise chirurgicale pour l'hémostase, 4 cas de paralysie récurrentielle unilatérale définitive et 3 cas d'hypoparathyroïdie transitoire. Par la même technique chirurgicale, Vignikin-Yéhouéssi et coll (VignikinYéhouéssi,2008) à Cotonou ont enregistré 6(0,67\%) décès, l'hypoparathyroidie définitive dans 5 cas $(0,57 \%)$, la paralysie récurrentielle 
unilatérale définitive dans 4 cas $(0,45 \%)$, les récidives : 12 cas $(1,35 \%)$ et les cicatrices chéloides: 21cas (2,36\%). En Europe, Miccoli et coll (Miccoli,2006), après chirurgie endoscopique ont recensé $0,9 \%$ de parésie récurrentielle unilatérale, $0,8 \%$ de paralysie récurrentielle définitive, $3,6 \%$ et $0,3 \%$ respectivement d'hypoparathyroïdie transitoire et définitive. La paralysie récurrentielle définitive a été observée dans $1,2 \%$ des cas dans la série d'Aytac et coll (Aytac, 2005) et 0,38\% dans celle de Pisello et coll (Pisello, 2005).

\section{Conclusion}

Les thyroïdectomies sont des interventions fréquentes en ORL à l'hôpital national de Niamey. Sa principale indication est le goitre multinodulaire. Le geste chirurgical le plus exécuté reste la thyroidectomie partielle. La mortalité a été nulle et la morbidité faible.

\section{References:}

1. Monabéka HG, Ondzotto G, Peko J.F, Kibeke P, Bouenizabila E, Nsakala-Kibangou N. La pathologie thyroïdienne au Centre Hospitalier Universitaire de Brazzaville. Cahiers Santé 2005; 15(1) :37-40.

2. Sani R, Illo A, Hassanaly A, James Didier L. Résultats des thyroïdectomies à l'Hôpital National de Niamey. Etude prospective de 12 mois: A propos de 105 cas. Annales de l'Université Abdou Moumouni de Niamey, Tome X-A $2010: 121-128$.

3. Toure A, Diallo AT, Camara LM, Touré FB, Camara ND. La chirurgie thyroïdienne : expérience du service de chirurgie générale du CHU Ignace Deen de Conakry. Mali Médical 2006; TOME XXI ; $3: 23-27$.

4. Dieng M, Cissé M, Ndour MD, Konaté I, Touré AO, Ka O, Dia A, Touré CT. Indications et résultats des thyroïdectomies réalisées au sein d'un service de chirurgie générale. A propos de 402 patients opérés. Revue Africaine de Chirurgie et Spécialités 2010 ; 4(9) : 2427.

5. Ayaché S,Tramier B,Chatelain D, Mardyla N,Benhaim T,Strunski V. Evolution of the thyroid surgical treatment to the total thyroidectomy. Study of about 735 patients. Ann Otolaryngol Chir Cervicofac 2005; 122(3):127-133.

6. Spanknebel K, Chabot JA, DI Giorgi M, Cheung K, Curtly J, Allendorf J, LO Gerfo P. Thyroidectomy using monitored local or conventional general anesthesia: an analysis of outpatient surgery, outcome and cost in 1194 consecutive cases. World J Surg 2006; 30(5):813-824. 
7. AL-Jaradi M, Sallam A, Jabr H, Borda A, Decaussin-Petrucci M, Berger N.Prevalence of differentiated thyroid cancer in 810 cases of surgically treated goiter in Yemen. Ann Saudi Med 2005; 25(5):394397.

8. Vignikin-Yéhouéssi B, Flatin M, Vodouhè S-J, Hounkpè Y.Y.C, Médji A.L.P. Place de la thyroidectomie en pratique ORL au CNHU de Cotonou.Rev.CAMES-Série A 2008 ; 06 :37-41.

9. Aytac B, Karamercan A. Recurrent laryngeal nerve injury and preservation in thyroidectomy. Saudi Med J 2005; 26(11):1746-1749.

10. Hill AG, Mwangi I, Wagana L. Thyroid disease in a rural Kenya hospital. East Afr Med J 2004; 81(12):631-633.

11. CL Conessa, B Cissoko, M Faye. Les complications de la chirurgie thyroïdienne à l'Hôpital Principal de Dakar: A propos de 155 interventions. Méd D’Afr Noire 2000 ; 47(3) :157-160.

12. Sanogo ZZ, Koïta AK, Koumaré S, Saye Z, Keita S, Camara M et coll. Prise en charge chirurgicale des goitres hyperthyroïdiens à Bamako. Mali Médical 2012 ; TOME XXVII ; 2 : 1-4.

13. Miccoli P, Berti P, Frustaci GL, Ambrosini CE, Materazzi G. Videoassisted thyroidectomy: indications and results. Langenbecks Arch Surg 2006; 391(2):68-71.

14. Colak T, Akca T, Kanik A, Yapici O, Aydin S. Total versus subtotal thyroidectomy for the management of benign multinodular goiter in an endemic region. Anz.J Surg 2004; 74(11):974-978.

15. Lubrena D, Levy-Chazal P, Araya Y, Avisse C. La recherche du nerf laryngé inférieur ou récurrent lors d'une lobectomie thyroïdienne. Ann Chir 2002; 127: 690- 94.

16. Pisello F, Géraci G, Sciume C, LI Volsi F, Facella T, Modica G. Prevention of complications in Thyroid Surgery recurrent laryngeal nerve injury personal experience on 313 cases. Ann Ital Chir 2005; 76(1):23-28. 